\title{
MAS: SISTEMA DE ATENCIÓN MÉDICA. Beneficios para estudiantes de la materia Ingeniería de Software y la comunidad con una aplicación móvil de servicios médicos
}

MAS: Medical Attention System. Benefits for students of Software engineering's matter and the Community with the Development of a Mobile

Application of Medical Services

MAIS: SISTEMA DE ASSISTÊNCIA MÉDICA. Benefícios para alunos da disciplina Engenharia de Software e comunidade com aplicativo mobile de atendimento médico Mario Martínez García Universidad de Guadalajara, México mmgmagma@hotmail.com https://orcid.org/0000-0002-5816-8434

José Roberto Muñoz Reyes

Universidad de Guadalajara, México Robertomrr1@hotmail.com https://orcid.org/0000-0001-6276-9235

Ricardo Pérez Zúñiga*

Universidad de Guadalajara, México perezuniga@udgvirtual.udg.mx https://orcid.org/0000-0001-9377-6083

Silvia Ramos Cabral

Universidad de Guadalajara, México Chivia06@hotmail.com https://orcid.org/0000-0003-4204-1700 
*Autor de correspondencia: Ricardo Pérez Zúñiga

\section{Resumen}

En el presente trabajo se presenta el desarrollo de una aplicación denominada sistema de atención médica, la cual fue creada para procurar reducir el tiempo de atención, costos y traslado del paciente desde su hogar o lugar de trabajo. En tal sentido, el presente artículo muestra el diseño de dicha aplicación para los sistemas operativos Android e iOS. La aplicación tiene un directorio telefónico con números de emergencia, laboratorios e instituciones de salud. Usando la geolocalización, se muestra a los usuarios cuáles médicos se encuentran cerca de su ubicación, en un radio de cinco kilómetros. Asimismo, ofrece un servicio de mensajes asíncronos entre pacientes y médicos. A través de la aplicación, el usuario puede acceder a su receta y descargarla una vez que haya realizado el pago de su consulta.

Para validar la implementación se trabajó con una muestra diagnóstica de 50 participantes, de los cuales $60 \%$ invierte hasta cuatro horas para su atención médica, mientras que $50 \%$ invierte dos salarios mínimos entre la consulta y el traslado. La exploración también demostró que más de 80 \% usaría una aplicación móvil para recibir atención médica. En el estudio de usabilidad, ocho preguntas fueron utilizadas con una escala de Liker. Los participantes quedaron satisfechos con la usabilidad de la aplicación, lo que resulta en un conjunto de $85 \%$ con respuestas fácil y muy fácil de utilizar.

El sistema de atención médica se desarrolló para proporcionar a los usuarios nuevas herramientas tecnológicas. Se demostró que esta aplicación reduce significativamente el tiempo dedicado al proceso de atención médica, así como los recursos económicos que se deben invertir en ello.

Palabras clave: aplicaciones móviles, conectividad, consulta médica, Ingeniería de Software. 


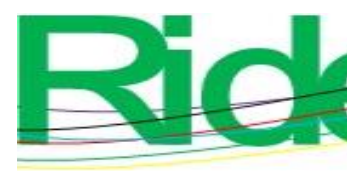

Revista Iberoamericana para la Investigación y el Desarrollo Educativo ISSN 2007-7467

assíncronas entre pacientes e médicos. Por meio do aplicativo, o usuário pode acessar a receita e fazer o download após efetuar o pagamento da consulta.

Para validar a implantação, trabalhamos com uma amostra diagnóstica de 50 participantes, dos quais $60 \%$ investem até quatro horas no atendimento médico, enquanto $50 \%$ investem dois salários mínimos entre a consulta e a transferência. A varredura também mostrou que mais de $80 \%$ usariam um aplicativo móvel para receber cuidados médicos. No estudo de usabilidade, foram utilizadas oito questões com uma escala de liker. Os participantes ficaram satisfeitos com a usabilidade do aplicativo, resultando em um conjunto de $85 \%$ com respostas fáceis e muito fáceis de usar.

O sistema de saúde foi desenvolvido para fornecer aos usuários novas ferramentas tecnológicas. Esta aplicação tem demonstrado reduzir significativamente o tempo despendido no processo de saúde, bem como os recursos financeiros que devem ser investidos nele.

Palavras-chave: aplicativos móveis, conectividade, consulta médica, Engenharia de software.

Fecha Recepción: Junio 2020

Fecha Aceptación: Octubre 2020

\section{Introducción}

En la actualidad, las aplicaciones móviles representan una oportunidad para los usuarios porque permiten reducir tiempos, costos, agilizar la comunicación y poner al alcance servicios que pueden mejorar nuestro nivel de vida (Álvarez y Fernández, 2020; Brandt et al., 2019; Pérez-Jover, Sala-González, Guilabert y Mira, 2019).

En México, tenemos la oportunidad de aportar para mejorar nuestras instituciones y en específico los centros de atención de la salud, de ahí que se deba aprovechar la tecnología para coadyuvar y alcanzar un sistema de salud de primer mundo (Balakrishnan et al., 2019; Gill et al., 2019).

Según datos del estudio de App Annie, en el primer trimestre de 2018 las descargas de iOS y Google Play crecieron más de $10 \%$ en comparación con el primer trimestre de 2017, y alcanzaron los 27500 millones de descargas, la cifra más alta hasta la fecha. Además, los usuarios de smartphone (combinados iOS y Google Play) consumieron $22 \%$ más en comparación con el primer trimestre de 2017, hasta alcanzar los 18400 millones de dólares, también un número récord (Blanco, 12 de abril de 2018). 

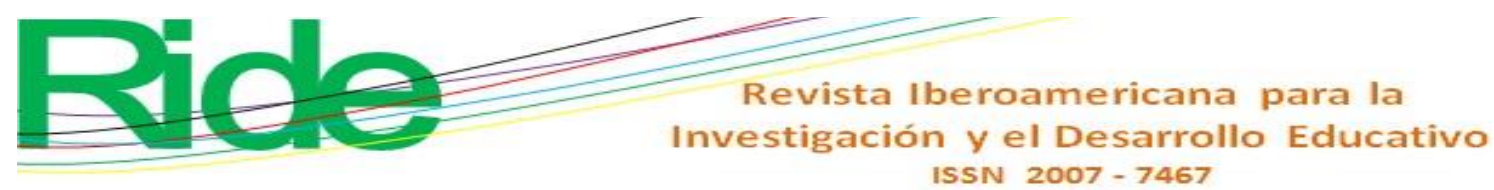

Las descargas de Google Play, comparando el intervalo anual entre trimestres, se vieron especialmente beneficiadas por mercados como India, Indonesia y Brasil, y el crecimiento de las descargas de iOS vino principalmente de EE. UU., Rusia y Turquía (Blanco, 12 de abril de 2018).

Cada día surgen nuevos desarrollos de aplicaciones móviles para buscar soluciones a problemas de alguna área en específico, entre ellos podemos destacar algunas desarrolladas para brindar servicios en el ámbito de salud, en áreas psicológicas, de nutrición, dermatológicas y médicas (Alessa, Hawley, Hock y de Witte, 2019; Chávez y Pozos, 2016; Pacheco y Krohling, 2020).

Ahora bien, un dispositivo móvil se puede definir como un aparato de tamaño pequeño, con capacidad para procesar información, con conexión permanente o intermitente a una red, con memoria limitada, que ha sido diseñado específicamente para una función (Baz, Ferreira, Álvarez y García, 2011), mientras que las aplicaciones móviles son programas de software diseñados para utilizarse en teléfonos inteligentes, tabletas y otros dispositivos móviles, cuyo propósito es realizar una tarea específica, a menudo básica, de rápido y fácil uso para el usuario común no avanzado (Morillo, 2011).

Existen varios métodos y lenguajes que tienen diferentes características y limitaciones en el desarrollo de aplicaciones (Längkvist, Jendeberg, Thunberg, Loutfi y Lidén, 2018). El desarrollo de las aplicaciones nativas se basa en el software que brinda cada sistema operativo a los programadores, llamado Software Development Kit o SDK: Android (Gironés, 2019), iOS (Baz et al., 2011) y Windows Phone (Amaya, 2015; Cuello y Vittone, 2013).

Para las aplicaciones web, su lenguaje de programación es HTML, JavaScript y CSS, y para el desarrollo de este tipo de aplicaciones no es necesario emplear un SDK. El desarrollo de las aplicaciones híbridas es parecido a una aplicación web, utilizando HTML, CSS y JavaScript (Ceballos, 2015). Las aplicaciones híbridas nos permiten con un mismo código obtener el software para diferentes plataformas como Android e iOS (Cuello y Vittone, 2013).

En entornos de software se propone una alternativa para optimizar los mecanismos de la atención médica, utilizando la tecnología y acercando los servicios médicos a través de las tecnologías de información y comunicación. Cualquier usuario que cuente con un dispositivo móvil y tenga acceso a internet puede emplear aplicaciones que contribuyen a la atención médica con servicios de calidad. Estos servicios buscan reducir tiempos de atención, costos 


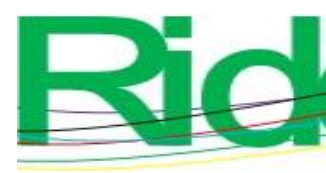

Revista Iberoamericana para la Investigación y el Desarrollo Educativo ISSN $2007-7467$

y largas esperas, eliminando la necesidad de recorrer grandes distancias, acercando a usuarios y médicos con la comunicación síncrona y asíncrona (Lunde, Nilsson, Bergland y Bye, 2019; Stevens, van der Sande, Beijer, Gerritsen, y Assendelft, 2019).

De acuerdo con la Organización Mundial de la Salud (OMS), las aplicaciones desarrolladas para mejorar la salud incluyen el comportamiento, bienestar y calidad de vida (Gazdecki, 2016). De hecho, según el reporte The mobile health global market report 20132017: the Commercialization of mHealth apps, 30 \% de las aplicaciones de salud móvil están destinadas para auxiliar a los profesionistas de la salud y $70 \%$ para los pacientes (Jahns, 2019).

Con el desarrollo de esta aplicación móvil (Sistema de Atención Médica, en adelante MAS), se ofrece una plataforma de servicios especializados que genera trabajo y atención médica, en la búsqueda de la reducción de tiempos de atención, costos y traslados. La aplicación brinda servicios que pueden considerarse como enfermedades básicas (entre ellas gripes, alergias, dolores musculares, entre otros). De esta manera, el médico que atienda al paciente tendrá la decisión de notificarle si requiere ser atendido físicamente o por medio de la aplicación, con lo cual se busca que los usuarios no pierdan mucho tiempo en asistir a las instituciones que brindan dichos servicios, ya que por lo general los pacientes solo sufren síntomas menores que pueden ser atendidos sin que tengan que presentarse físicamente al lugar.

\section{Objetivos}

Esta aplicación fue diseñada e implementada para permitir la atención médica en línea cuando las situaciones fueran consideradas de primer nivel, y cuenta con una base de datos para gestionar el intercambio de información. Nuestra hipótesis es que al usar esta aplicación los usuarios reducirán el tiempo y los costos en sus consultas.

Por otra parte, se evaluó la usabilidad y tomamos en cuenta los costos para los usuarios en sus consultas y el tiempo invertido en ir al consultorio del médico usando los métodos tradicionales. Finalmente, la aceptabilidad de una aplicación con estas características fue consultada con usuarios potenciales con una muestra de 50 participantes en la Región Valles, en el estado de Jalisco (México). 


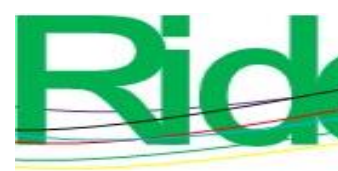

Revista Iberoamericana para la Investigación y el Desarrollo Educativo ISSN $2007-7467$

\section{Metodología de desarrollo}

Para el desarrollo de este proyecto la metodología de ingeniería de software se dividió en cuatro etapas. Primera etapa: investigación de aplicaciones existentes en el mercado y consulta ciudadana sobre la factibilidad y viabilidad de una aplicación con las características de la que se desarrolló. Segunda etapa: recolección de requisitos y desarrollo del sistema. Tercera etapa: implementación. Cuarta etapa: pruebas del sistema y análisis de resultados (Sommerville, 2011).

En la primera etapa, se realizó una investigación sobre aplicaciones similares, de las cuales se encontraron las siguientes:

- Doctoralia: Diseñada para la gestión de citas médicas, además de que permite encontrar especialistas del seguro contratado por los pacientes (Llordachs, 2020).

- American Well: Ofrece consultas médicas en línea, servicios psicológicos y nutricionistas. Spruce brinda videoconsultas para padecimientos dermatológicos (Schoenberg y Schoenberg, 2020).

- MediQó: Ofrece consultas a través de geolocalización, donde el médico acude al domicilio o área laboral del paciente para brindar la atención requerida (García y Cervantes, 2020).

- Heal: Brinda consultas personalizadas a domicilio para los pacientes que requieran un médico (Dua, Desai y Drobnick, 2020).

Sobre la consulta ciudadana, se tomó una muestra y se aplicó la encuesta en la Región Valles del estado de Jalisco, en la cual destacan las aportaciones que se muestran en las tablas 1 y 2 , y figuras 11 y 12, en la sección de evaluación de las pruebas del sistema y resultados.

La segunda etapa, para la recolección de requisitos que se han definido para el sistema, se dividió en dos partes: en la primera se analizaron los requisitos de aplicaciones existentes y se identificaron oportunidades de mejoras; en la segunda se recabaron clientes potenciales definidos como usuario-paciente y usuario-médico; esto para mejorar la experiencia e interacción de la app en los usuarios y médicos (Pressman, 2010).

Las metodologías empleadas para el desarrollo del sistema son modelo por prototipos (Cuello y Vittone, 2013). Este ha sido seleccionado para la recolección de requisitos del sistema, el análisis del sistema, además de la aplicación del lenguaje de modelo unificado (Unified Modeling Language, UML) con la creación de los diagramas de casos de uso, clases 


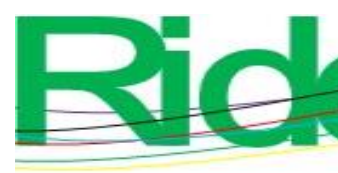

Revista Iberoamericana para la Investigación y el Desarrollo Educativo ISSN 2007-7467

y entidad relación; otra técnica utilizada fue el modelo en cascada (Pressman, 2010) para el desarrollo de las etapas, diseño del sistema, implementación y pruebas.

En la tercera etapa, una vez establecida y definida la estructura del MAS, se inició la fase de implementación utilizando PhoneGap para el desarrollo de la app, así como lenguajes de programación, tales como HTML, CSS, JavaScript, jQuery y PHP, los cuales nos permiten crear una app nativa, optimizando tiempo en desarrollo de código, ya que nos brinda el poder obtener una aplicación para diferentes sistemas operativos Android e iOS.

En la cuarta etapa, para la evaluación de pruebas, se diseñó un plan para probar cada elemento de manera funcional; se probaron los módulos de acceso a usuarios, solicitar cita médica, citas activadas, citas programadas, historial de citas, configuración, diccionario de negocios, publicidad y la funcionalidad de la API de Google Maps de Android e iOS con la geolocalización de cada dispositivo móvil. Para conocer si una prueba era exitosa o presentaba errores, se establecieron dos criterios de aceptación:

Criterio exitoso. Esta fase de la prueba fue considerada exitosa cuando fueron probados 99 \% de casos de uso; para ello, se consideró la relación existente entre las entradas validadas, las anomalías encontradas y los resultados esperados.

Criterio suspendido. En esta fase se consideró que una prueba debía ser suspendida cuando existían errores que no permitían al sistema continuar con la ejecución de un proceso de prueba. Por eso, se consideró esencial corregir el error antes de continuar con los siguientes procesos de prueba.

\section{Diseño e implementación del sistema}

El proyecto MAS plantea el desarrollo de una aplicación móvil para sistemas operativos Android e iOS, teniendo como objetivo diseñar e implementar una aplicación móvil que permita la atención médica en línea o personalizada en pacientes con enfermedades de grado básico a través de un dispositivo móvil.

La figura 1 muestra la arquitectura del sistema, que consiste en la implementación de un servidor para el envío de información de los usuarios paciente y usuarios médicos, además de poder generar consultas en el servidor de la base de datos. 


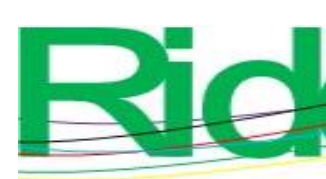

Revista Iberoamericana para la Investigación y el Desarrollo Educativo ISSN 2007-7467

Figura 1. Interfaz de la arquitectura del sistema MAS

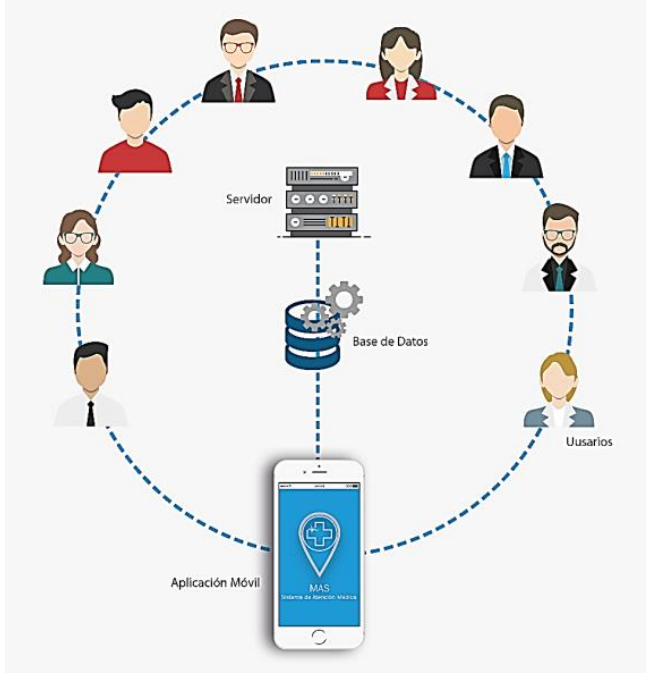

Fuente: Elaboración propia

La aplicación del sistema MAS permite a los usuarios-pacientes, a través de geolocalización, encontrar médicos disponibles para brindar una atención o consulta médica. La figura 2 enseña la interfaz de inicio de sesión y se divide en dos partes: la primera consta del fondo azul en plano y el logotipo establecido para el sistema; la segunda parte contiene los campos para que el usuario-paciente y el usuario-médico introduzcan su nombre de usuario y contraseña para poder ser autenticados en la aplicación móvil.

Figura 2. Aplicación MAS (pantalla principal)

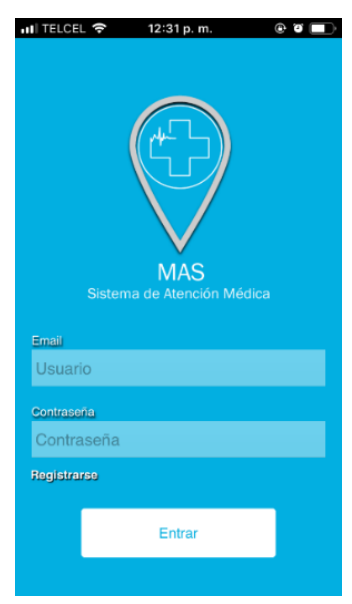

Fuente: Elaboración propia 


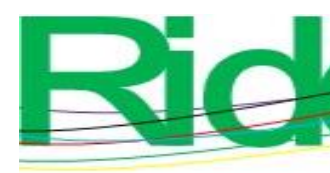

Revista Iberoamericana para la
Investigación y el Desarrollo Educativo
ISSN $2007-7467$

La figura 3 muestra la interfaz apariencia principal y se divide en dos partes: la primera muestra un banner de publicidad donde podrá visualizar opciones de negocios; la segunda parte enseña un menú donde el usuario-paciente puede solicitar una cita médica, consultar sus citas programadas, revisar su historial de atención y actualizar su información personal.

Figura 3. Aplicación MAS (pantalla principal para el usuario-paciente)

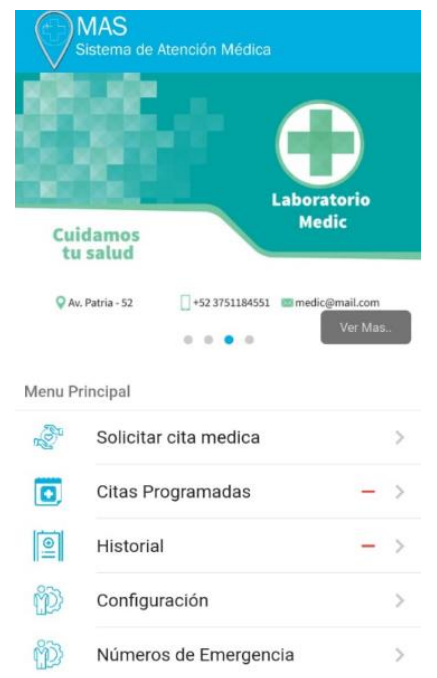

Fuente: Elaboración propia

La figura 4 muestra la interfaz para solicitar cita y se ha estructurado para que el usuario-paciente pueda identificar los médicos más cercanos a su domicilio por medio de geolocalización. Una vez que el paciente selecciona al médico más cercano podrá entablar una conversación de atención por medio de un chat para ser valorado en consultorio o directamente en su domicilio. 

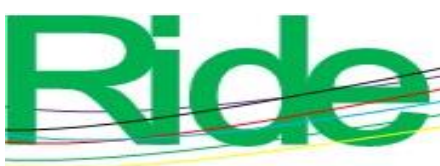

Revista Iberoamericana para la Investigación y el Desarrollo Educativo ISSN $2007-7467$

Figura 4. Aplicación MAS (pantalla para que el usuario-paciente solicite una cita médica)

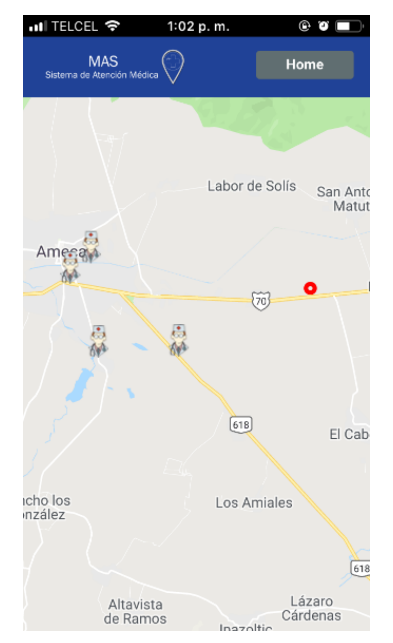

Fuente: Elaboración propia

La figura 5 muestra la interfaz para citas programadas; el usuario podrá consultar sus citas programadas de consultas médicas y agendar una cita con su médico particular o cualquier otro médico de la app.

Figura 5. Aplicación MAS (pantalla citas médicas programadas por el usuario-paciente)

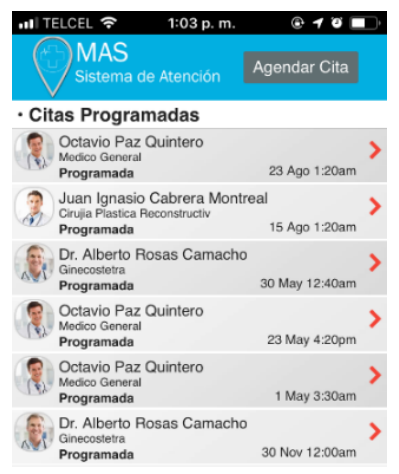

\section{$\leftarrow$ กิ}

Fuente: Elaboración propia

La figura 6 muestra la interfaz historial de citas médicas; el usuario podrá visualizar su historial de consultas realizadas o canceladas en tiempo real. 


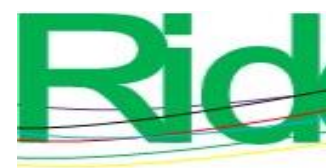

Revista Iberoamericana para la
vestigación y el Desarrollo Educativo
ISSN $2007-7467$

Figura 6. Aplicación MAS (pantalla historial citas médicas del usuario-paciente)

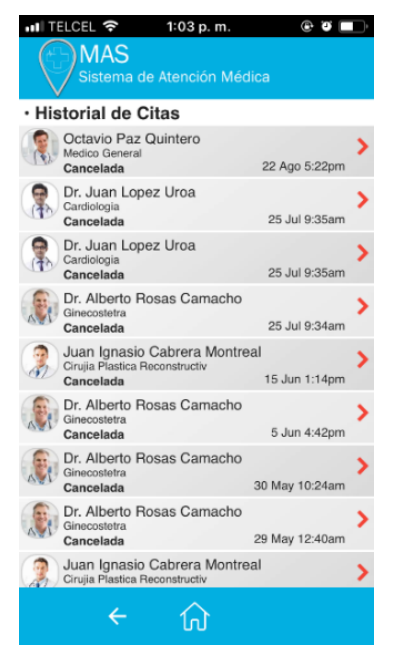

Fuente: Elaboración propia

\section{La aplicación usuario-paciente}

Esta muestra la información almacenada en el servidor de la base de datos de médicos registrados, es decir, la búsqueda de médicos por los usuarios pacientes por medio de la geolocalización, lo que permite visualizar médicos disponibles para brindar una consulta en un radio aproximado de cinco kilómetros a partir de la ubicación de cada usuario-paciente.

\section{La aplicación usuario-médico}

Esta permite la comunicación de manera directa entre el usuario-paciente y el usuariomédico a través de un chat que se activa una vez que existe una alerta de consulta por parte de los usuarios pacientes.

\section{Evaluación de las pruebas del sistema y resultados}

Para cumplir con la verificación del funcionamiento correcto del MAS, se desarrollaron diferentes tipos de pruebas que cumplen con cada una de las funciones del sistema. Para cada caso de función se elaboró un tipo de prueba. 


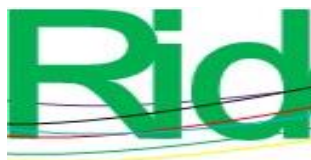

Revista Iberoamericana para la
Investigación y el Desarrollo Educativo
ISSN $2007-7467$

\section{Pruebas de configuración}

La figura 7 muestra el resultado de la prueba, donde se validó que los usuariospacientes y los usuarios-médicos puedan actualizar su información personal requerida por el sistema.

Figura 7. Aplicación MAS (pantalla configuración de contraseña)

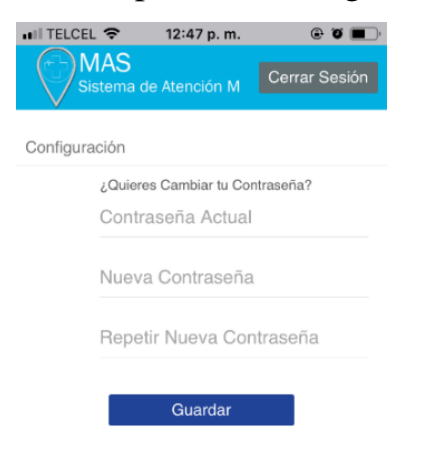

$\leftarrow$ ते

Fuente: Elaboración propia

\section{Pruebas de geolocalización}

La figura 8 muestra el resultado de la prueba de validación sobre la ubicación del usuario-paciente, así como la visualización de los médicos cercanos en un área de cinco kilómetros. 


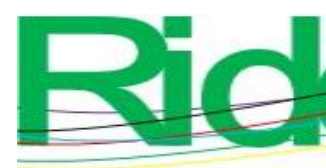

Revista Iberoamericana para la Investigación y el Desarrollo Educativo ISSN $2007-7467$

Figura 8. Aplicación MAS (pantalla geolocalización)

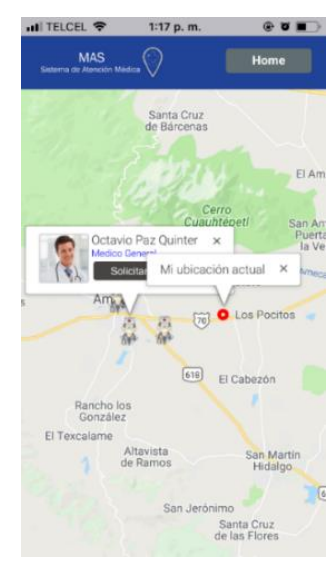

Fuente: Elaboración propia

Además de comprobar el cambio de estado del botón solicitar cita médica (como se muestra en la figura 3), una vez que el usuario solicita una cita, el botón cambia a cita activada, como se muestra en la figura 9.

Figura 9. Aplicación MAS (pantalla menú cita médica)
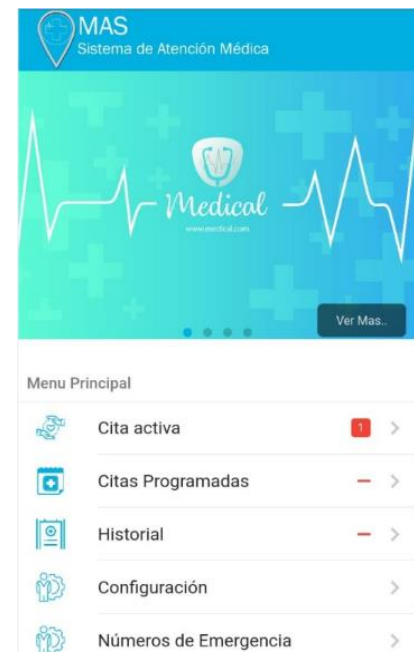

Fuente: Elaboración propia

\section{Pruebas de integración}

La figura 10 muestra el resultado de la prueba interfaz usuario-paciente, donde se evidencia la comunicación por medio del chat al momento de contactarse el paciente con el médico, así como las pruebas de estado en la solicitud de atención médica. 
Figura 10. Aplicación MAS (pantalla chat usuario-paciente)

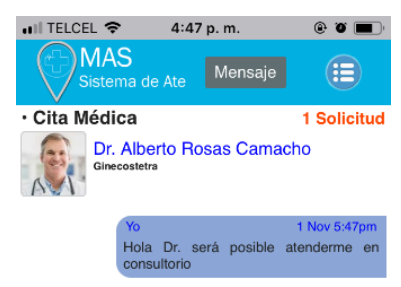

\section{$\leftarrow$ 介}

Fuente: Elaboración propia

\section{Resultados}

Al aplicar la encuesta a los posibles usuarios de la aplicación sobre su percepción en la atención médica en la Región Valles del estado de Jalisco (México), encontramos que más de $60 \%$ opina que el servicio es malo, como se muestra en la tabla 1.

Tabla 1. Percepción de la atención médica

\begin{tabular}{|l|c|c|}
\hline & Buena & Mala \\
\hline $\begin{array}{l}\text { En general, ¿cómo califica a } \\
\text { las instituciones que brindan } \\
\text { servicios de salud? }\end{array}$ & $32 \%$ & $68 \%$ \\
\hline $\begin{array}{l}\text { Considera que el tiempo } \\
\text { que hay entre consulta } \\
\text { médica es... }\end{array}$ & $38 \%$ & $62 \%$ \\
\hline $\begin{array}{l}\text { Considera que la calidad de } \\
\text { atención que le brinda el } \\
\text { personal de salud es... }\end{array}$ & $44 \%$ & $66 \%$ \\
\hline
\end{tabular}

Fuente: Elaboración propia 

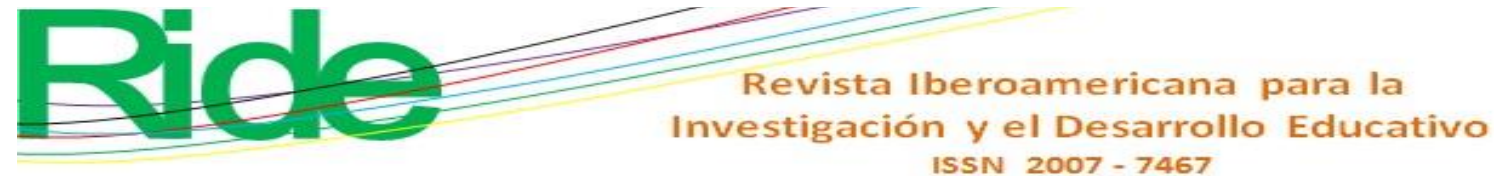

Respecto a los tiempos de atención y traslados que los usuarios del servicio médico invierten para su atención médica (básica o primer nivel en la Región Valles del estado Jalisco, México), detectamos que más de $60 \%$ de la muestra invierte hasta cuatro horas para su atención médica (entre traslado y tiempo de consulta, como se aprecia en la figura 11).

Figura 11. Tiempo invertido en la atención médica

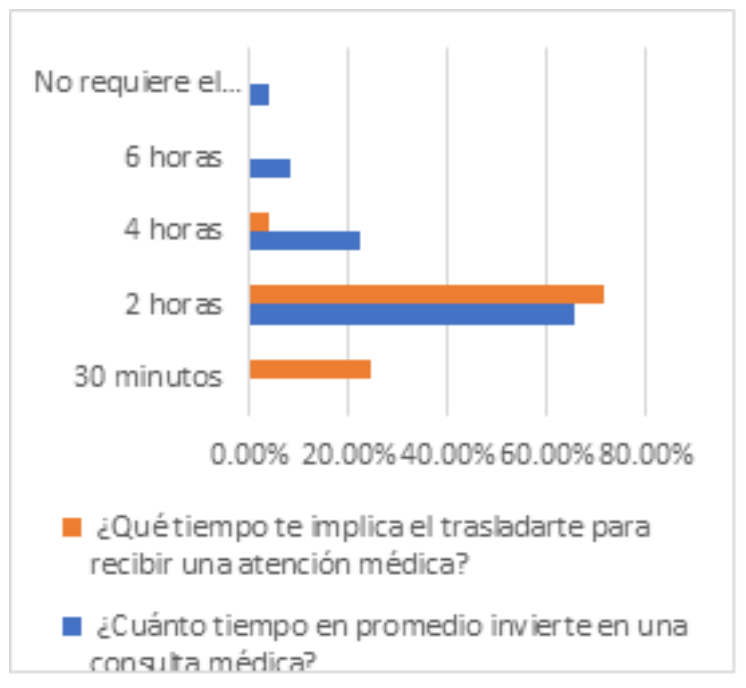

Fuente: Elaboración propia

Nos damos cuenta de que en la actualidad y en pleno siglo XXI existen más personas con dispositivos móviles que con internet en la Región Valles del Estado de Jalisco (México), y que más de 80 \% utilizaría una aplicación móvil para recibir atención médica (como se muestra en la tabla 2). 
Tabla 2. Disponibilidad para utilizar la aplicación MAS

\begin{tabular}{|l|c|c|}
\hline & Sí & No \\
\hline $\begin{array}{l}\text { ¿Dispone de un dispositivo } \\
\text { móvil inteligente? }\end{array}$ & $96 \%$ & $4 \%$ \\
\hline $\begin{array}{l}\text { ¿Cuenta usted con acceso a } \\
\text { internet? }\end{array}$ & $92 \%$ & $8 \%$ \\
\hline $\begin{array}{l}\text { ¿Utilizaría una aplicación } \\
\text { móvil para recibir una } \\
\text { consulta médica } \\
\text { especializada desde su } \\
\text { teléfono, que cuente con } \\
\text { médicos a su alrededor? }\end{array}$ & $64 \%$ & $36 \%$ \\
\hline $\begin{array}{l}\text { ¿Le gustaría recibir una } \\
\text { consulta médica } \\
\text { directamente en su } \\
\text { domicilio? }\end{array}$ & & \\
\hline $\begin{array}{l}\text { ¿Utilizaría una aplicación } \\
\text { móvil que le ahorre tiempo } \\
\text { de traslado para recibir una } \\
\text { atención médica? }\end{array}$ & & \\
\hline $\begin{array}{l}\text { ¿Utilizaría una aplicación } \\
\text { que le brinde atención } \\
\text { médica las 24 horas en } \\
\text { línea? }\end{array}$ & & \\
\hline $\begin{array}{l}\text { ¿Utilizaría una aplicación } \\
\text { que le brinde atención } \\
\text { médica las 24 horas } \\
\text { directamente en su } \\
\text { domicilio? }\end{array}$ & & \\
\hline $\begin{array}{l}\text { ¿Utilizaría una aplicación } \\
\text { móvil que le brinde }\end{array}$ & & \\
\hline
\end{tabular}




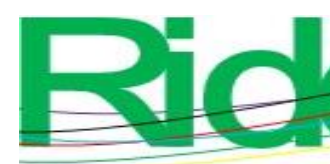

Revista Iberoamericana para la Investigación y el Desarrollo Educativo ISSN 2007 - 7467

\begin{tabular}{|l|c|c|}
\hline $\begin{array}{l}\text { servicios para solicitar } \\
\text { ambulancia? }\end{array}$ & \\
\hline ¿Utilizaría una aplicación & $92 \%$ & $8 \%$ \\
móvil que le brinde & \\
servicios de contacto de & & \\
laboratorio clínico? & & \\
\hline
\end{tabular}

Fuente: Elaboración propia

Por otra parte, $50 \%$ de la muestra comenta que invierte entre consulta y traslado dos salarios mínimos, más el día que deben usar para su atención. Esto significa que deben emplear dos salarios mínimos, además de los salarios que ganan en una jornada laboral, como se aprecia en la figura 12.

Figura 12. Recursos invertidos para recibir atención médica

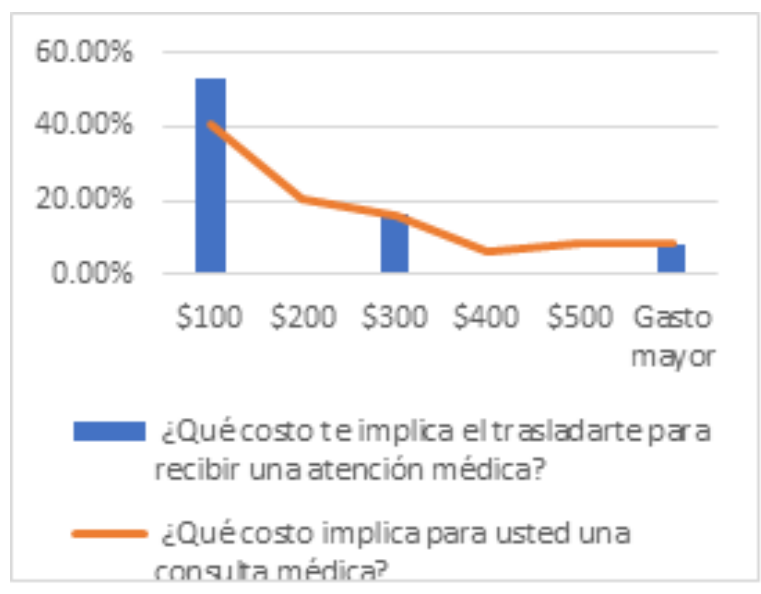

Fuente: Elaboración propia

Para el registro de la percepción usabilidad se empleó una encuesta con ocho reactivos, utilizando una escala de Liker con un rango de entre 1 (muy fácil) y 5 (muy difícil). La encuesta identifica el nivel de usabilidad percibido de $1 \%$ a $100 \%$. En la tabla 3 se aprecian los resultados obtenidos de la prueba de usabilidad y la ponderación obtenida. 


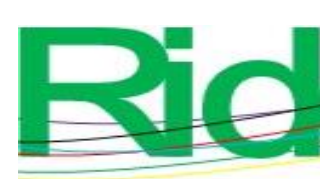

Revista Iberoamericana para la Investigación y el Desarrollo Educativo ISSN 2007 - 7467

Tabla 3. Resultado de usabilidad

\begin{tabular}{|c|c|c|c|c|c|}
\hline PREGUNTA & Muy fácil & Fácil & Normal & Difícil & Muy difícil \\
\hline $\begin{array}{l}\text { 1.- } \\
\text { Identifíquese } \\
\text { en la } \\
\text { aplicación, } \\
\text { utilizando su } \\
\text { usuario. }\end{array}$ & 100 & 100 & & & \\
\hline $\begin{array}{l}\text { 2.- Solicite } \\
\text { una cita } \\
\text { médica y } \\
\text { seleccione el } \\
\text { médico que } \\
\text { prefiera. }\end{array}$ & & 90 & 10 & & \\
\hline $\begin{array}{l}\text { 3.- Entable } \\
\text { una } \\
\text { conversación } \\
\text { con dicho } \\
\text { médico. }\end{array}$ & & 70 & 30 & & \\
\hline $\begin{array}{l}\text { 4.- Agende } \\
\text { una cita } \\
\text { médica. }\end{array}$ & & 80 & 20 & & \\
\hline $\begin{array}{l}\text { 5.- Consulte } \\
\text { sus citas } \\
\text { médicas a } \\
\text { través de su } \\
\text { historial. }\end{array}$ & & 80 & 20 & & \\
\hline $\begin{array}{l}\text { 6.- Cambie } \\
\text { la contraseña } \\
\text { actual por }\end{array}$ & & 100 & & & \\
\hline
\end{tabular}



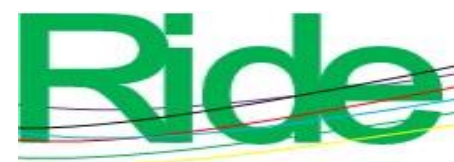

Revista Iberoamericana para la Investigación y el Desarrollo Educativo

ISSN 2007 - 7467

permitan estar a la altura de los países más desarrollados; de lo contrario, nos veremos inmersos en el consumismo de tecnología extranjera (Santamaría y Hernández, 2015).

\section{Comparación con trabajos previos}

Las funciones de la aplicación desarrollada se compararon con otras que existen en el mercado. Un ejemplo es Doctoralia, que ofrece una base de datos de médicos para programar una cita con el médico más cercano y según su especialidad. Por otra parte, American Well solo brinda servicios de atención médica centrados en las áreas de nutrición y psicología; además, en este caso los médicos pueden interactuar con los usuarios a través de videoconsultas para sugerir soluciones especializadas, mientras que Spruce centra su atención en usuarios con problemas dermatológicos básicos a través de videoconsulta.

La aplicación presentada en este artículo se comparó con otras tres aplicaciones, las cuales si bien son prácticas y funcionales, tienen servicios limitados en comparación con los propuestos en MAS (tabla 4). 
Tabla 4. Tabla comparativa de servicios y características entre Heal, MediQó y la aplicación desarrollada

\begin{tabular}{|c|c|c|c|}
\hline $\begin{array}{l}\text { Características y } \\
\text { funciones } \\
\text { disponibles en la } \\
\text { aplicación. }\end{array}$ & MediQó & Heal & MAS \\
\hline $\begin{array}{l}\text { Consulta a } \\
\text { domicilio. }\end{array}$ & $\mathrm{X}$ & $\mathrm{X}$ & $\mathrm{X}$ \\
\hline $\begin{array}{l}\text { Información } \\
\text { personal del médico. }\end{array}$ & $\mathrm{X}$ & $\mathrm{X}$ & $\mathrm{X}$ \\
\hline $\begin{array}{l}\text { Información } \\
\text { personal del } \\
\text { paciente. }\end{array}$ & $X$ & $X$ & $\mathrm{X}$ \\
\hline $\begin{array}{l}\text { Programe una cita y } \\
\text { reciba notificación } \\
30 \text { minutos antes. }\end{array}$ & $\mathrm{X}$ & & $\mathrm{x}$ \\
\hline $\begin{array}{l}\text { Historia clínica del } \\
\text { paciente. }\end{array}$ & $X$ & & $\mathrm{x}$ \\
\hline Kit de medicina. & $\mathrm{X}$ & $\mathrm{X}$ & $\mathrm{x}$ \\
\hline $\begin{array}{l}\text { Entrega de } \\
\text { medicamentos a } \\
\text { domicilio. }\end{array}$ & $\mathrm{X}$ & & $\mathrm{x}$ \\
\hline $\begin{array}{l}\text { Receta o aplicación } \\
\text { escrita. }\end{array}$ & $\mathrm{X}$ & $\mathrm{X}$ & $\mathrm{x}$ \\
\hline $\begin{array}{l}\text { Servicio de } \\
\text { ambulancia. }\end{array}$ & $\mathrm{X}$ & & $\mathrm{x}$ \\
\hline Geolocalización. & $\mathrm{X}$ & $\mathrm{X}$ & $\mathrm{x}$ \\
\hline Chat. & $\mathrm{X}$ & & $\mathrm{x}$ \\
\hline Videoconsulta. & & & $\mathrm{x}$ \\
\hline Servicios en línea. & $\mathrm{X}$ & $\mathrm{X}$ & $\mathrm{x}$ \\
\hline $\begin{array}{l}\text { Servicio de } \\
\text { recomendación. }\end{array}$ & & & $\mathrm{x}$ \\
\hline
\end{tabular}

Fuente: Elaboración propia 


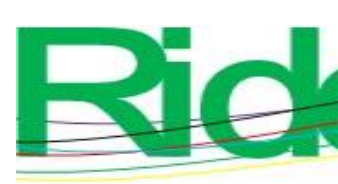

Revista Iberoamericana para la Investigación y el Desarrollo Educativo ISSN $2007-7467$

\section{Conclusiones y trabajos futuros}

En este proyecto se presentan los resultados del desarrollo del sistema de atención médica construido para brindar a los usuarios nuevas herramientas tecnológicas que faciliten el obtener una consulta médica sin necesidad de invertir demasiado tiempo ni recursos en la atención de enfermedades simples.

Con el uso de la aplicación MAS los pacientes ahorran tiempo y dinero, ya que la consulta es de 50 pesos, lo que reduce más de $75 \%$ del gasto invertido con los métodos tradicionales y sin perder la jornada laboral.

Por tanto, podemos concluir que la usabilidad del sistema MAS es aceptable al considerar la media en $85 \%$ en el rango de respuestas 1 y 2 (muy fácil y fácil). Aun así, identificamos aspectos que se pueden mejorar como los siguientes: habilitar un historial de salud por cada usuario, con niveles de glucosa, colesterol, triglicéridos, entre otros, que influyan en la salud; incorporar módulos para dar seguimiento a las condiciones físicas de los pacientes para recibir consejos médicos y sugerencias de hábitos para prevenir enfermedades; y agregar el histórico de vacunas que los usuarios han recibido.

A través de esta aplicación, un usuario puede solicitar una cita médica usando la geolocalización, la cual está integrada para mostrar cuáles médicos están cerca de su ubicación, en un radio de cinco kilómetros. Además, permite la interacción entre un usuario y un médico a través de un chat como primer acercamiento a distancia. De hecho, si el médico lo considera necesario, le indicará al usuario que vaya a su oficina o consultorio para una revisión personalizada. En este estudio, un médico comenzará a generar la prescripción médica en línea en caso de que la enfermedad lo requiera. Una vez que el usuario haya realizado el pago, puede descargar su receta y podrá comprar el medicamento.

Aplicaciones con características como las del sistema expuesto en este artículo ayudan a mantener una distancia saludable entre pacientes y médicos, reduciendo grandes grupos de pacientes que esperan en las clínicas o en los consultorios médicos, lo cual disminuye la posibilidad de contagios, como sucede con la covid-19.

Desarrollos como este deben ser analizados por estudiantes de las áreas de Ingeniería de Software, Algoritmos y aquellos que estudian en los Programas Educativos de Sistemas, Computación, Electrónica, Mecatrónica y maestrías afines para motivarlos a implementar sus propias innovaciones y/o mejorar la presente propuesta. 


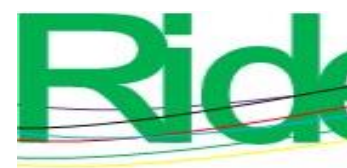

Revista Iberoamericana para la
Investigación y el Desarrollo Educativo
ISSN $2007-7467$

Asimismo, coincidimos con publicaciones e investigaciones en la cuales se subraya que el desarrollo de aplicaciones orientadas a fortalecer la atención médica modifican nuestras vidas, ya que les permiten a los médicos contar con mayores insumos para realizar mejores diagnósticos, mientras que a los pacientes les ofrece una mejor calidad de vida.

Finalmente, como trabajo futuro se considera la necesidad de incorporar módulos para dar seguimiento a los hábitos y condiciones físicas de los pacientes y correlacionarlos con su salud para prevenir enfermedades.

\section{Referencias}

Alessa, T., Hawley, M. S., Hock, E. S. and de Witte, L. (2019). Smartphone Apps to Support Self-Management of Hypertension: Review and Content Analysis. JMIR mHealth and uHealth, 7(5), e13645. Doi: https://doi.org/10.2196/13645

Álvarez, D. and Fernández, I. (2020). Addressing database variability in learning from medical data: An ensemble-based approach using convolutional neural networks and a case of study applied to automatic sleep scoring. Computers in Biology and Medicine, 119. Doi: https://doi.org/10.1016/j.compbiomed.2020.103697

Amaya, Y. D. (2015). Metodologías ágiles en el desarrollo de aplicaciones para dispositivos móviles. Estado actual. Revista de Tecnología,12(2), 111-123. Doi: https://doi.org/10.18270/rt.v12i2.1291

Balakrishnan, A. S., Nguyen, H. G., Shinohara, K., Au Yeung, R., Carroll, P. R. and Odisho, A. Y. (2019). A Mobile Health Intervention for Prostate Biopsy Patients Reduces Appointment Cancellations: Cohort Study.Journal of Medical Internet Research, 21(6), e14094. Doi: https://doi.org/10.2196/14094

Baz, A., Ferreira, I., Álvarez, M. y García, R. (2011). Dispositivos móviles. Recuperado de http://isa.uniovi.es/docencia/SIGC/pdf/telefonia_movil

Blanco, R. (12 de abril de 2018). El mundo de las descargas de iOS y Google Play. Forbes México. Recuperado de https://www.forbes.com.mx/el-mundo-de-las-apps/.

Brandt, L. R., Hidalgo, L., Diez-Canseco, F., Araya, R., Mohr, D. C., Menezes, P. R. and Miranda, J. J. (2019). Addressing Depression Comorbid With Diabetes or Hypertension in Resource-Poor Settings: A Qualitative Study About User Perception of a Nurse-Supported Smartphone App in Peru. JMIR Mental Health, 6(6), e11701. Doi: https://doi.org/10.2196/11701 

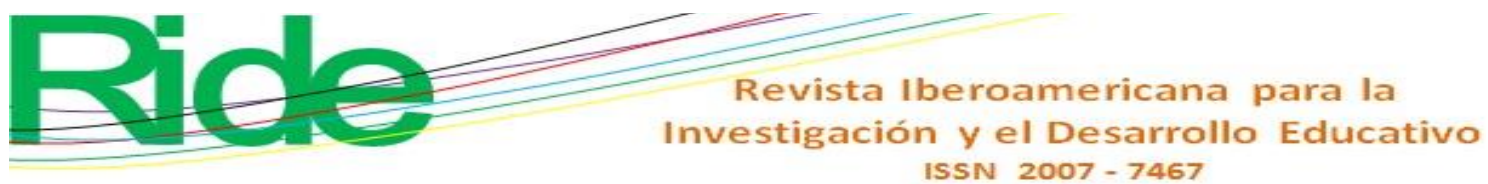

Ceballos, F. J. (2015). Java: interfaces gráficas y aplicaciones para internet (4. ${ }^{\mathrm{a}}$ ed.). Madrid, España: Ra-Ma.

Chávez, O. and Pozos, P. (2016). The Latin American laws of correct nutrition: Review, unified interpretation, model and tools. Computers in Biology and Medicine, 70, 6779. Doi: https://doi.org/10.1016/j.compbiomed.2015.12.019

Cuello, J. y Vittone, J. (2013). Diseñando apps para móviles (vol. 1.1). Recuperado de http://www.catedranaranja.com.ar/taller4/notas_T4/Disenando_apps_para_moviles_ CAP.5.pdf

Dua, R., Desai, N. y Drobnick, G. (2020). Heal (versión 4.21.1) [aplicación móvil]. Descargado de https://heal.com/ 24

García, H. y Cervantes, A. (2020). mediQó (versión 2.0.1) [aplicación móvil]. Descargado de https://mediqo.mx/

Gazdecki, A. (2016). 9 Mobile Technology Trends For 2017 (Infographic). Bizness Apps. Retrieved from https://www.biznessapps.com/blog/mobile-technology-trends/

Gill, R., Ogilvie, G., Norman, W. V., Fitzsimmons, B., Maher, C. and Renner, R. (2019). Feasibility and Acceptability of a Mobile Technology Intervention to Support Postabortion Care in British Columbia: Phase I. Journal of Medical Internet Research, 21(5), e13387. Doi: https://doi.org/10.2196/13387

Gironés, J. T. (2019). El gran libro de Android (7. ${ }^{a}$ ed.). Málaga, España: Marcombo S. A.

Jahns, R. G. (2019). The mobile health global market report 2013-2017.The commercialization of Mhealth applications $\left(3^{\text {th }}\right.$ ed.). Research2guidance. Retrieved from http://research2guidance.com/product/mobile-health-market-report-2013$2017 /$

Längkvist, M., Jendeberg, J., Thunberg, P., Loutfi, A. and Lidén, M. (2018). Computer aided detection of ureteral stones in thin slice computed tomography volumes using Convolutional Neural Networks. Computers in Biology and Medicine, 97, 153-160. Doi: https://doi.org/10.1016/j.compbiomed.2018.04.021

Llordachs, F. (2020). Doctoralia (versión 3.1.2) [aplicación móvil]. Descargado de https://www.doctoralia.com.mx/

Lunde, P., Nilsson, B. B., Bergland, A. and Bye, A. (2019). Feasibility of a Mobile Phone App to Promote Adherence to a Heart-Healthy Lifestyle: Single-Arm Study. JMIR Formative Research, 3(2), e12679. Doi: https://doi.org/10.2196/12679 


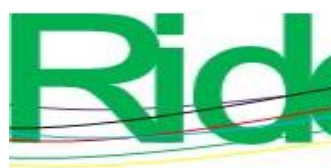

Revista Iberoamericana para la
Investigación y el Desarrollo Educativo
ISSN $2007-7467$

Morillo, J. D. (2011). Introducción a los dispositivos móviles. Recuperado de https://www.exabyteinformatica.com/uoc/Informatica/Tecnologia_y_desarrollo_en_ dispositivos_moviles/Tecnologia_y_desarrollo_en_dispositivos_moviles_(Modulo_ 2).pdf

Pacheco, A. G. C. and Krohling, R. A. (2020). The impact of patient clinical information on automated skin cancer detection. Computers in Biology and Medicine, 116, 103545. Doi: https://doi.org/10.1016/j.compbiomed.2019.103545

Pérez-Jover, V., Sala-González, M., Guilabert, M. and Mira, J. J. (2019). Mobile Apps for Increasing Treatment Adherence: Systematic Review. Journal of Medical Internet Research, 21(6), e12505. Doi: https://doi.org/10.2196/12505

Pressman, R. S. (2010). Software Engineering: A Practitioner's Approach [version PDF]. Retrieved from http://seu1.org/files/level4/IT242/Software\%20Engineering\%20_\%207th\%20Edition.pdf

Santamaría, G. y Hernández, E. (2015). Aplicaciones médicas móviles: definiciones, beneficios y riesgos. Salud Uninorte, 31(3), 599-607. Recuperado de https://www.redalyc.org/articulo.oa? $\mathrm{id}=817 / 81745378016$

Schoenberg, I. and Schoenberg, R. (2020). American Well (versión 12.0.815) [aplicación móvil]. Descargado de https://business.amwell.com/about-us

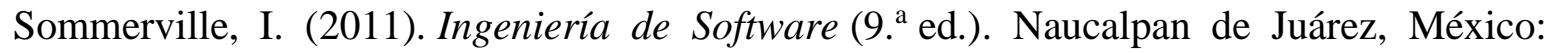
Pearson.

Stevens, W. J. M., van der Sande, R., Beijer, L. J., Gerritsen, M. G. and Assendelft, W. J. (2019). eHealth Apps Replacing or Complementing Health Care Contacts: Scoping Review on Adverse Effects. Journal of Medical Internet Research, 21(3), e10736. Doi: https://doi.org/10.2196/10736 


\begin{tabular}{|l|l|}
\hline Rol de Contribución & Autor (es) \\
\hline Conceptualización & Mario Martínez García \\
\hline Metodología & Ricardo Pérez Zúñiga \\
\hline Software & José Roberto Muñoz Reyes \\
\hline Validación & Silvia Ramos Cabral \\
\hline Análisis Formal & José Roberto Muñoz Reyes \\
\hline Investigación & Mario Martínez García \\
\hline Recursos & Mario Martínez García \\
\hline Curación de datos & José Roberto Muñoz Reyes \\
\hline Proyectos & Mario Martínez García \\
\hline $\begin{array}{l}\text { Adquisición de fondos } \\
\text { edición }\end{array}$ & Silvia Ramos Cabral \\
\hline Visualización & Mario Martínez García \\
\hline
\end{tabular}

